\title{
A Distributed Opportunistic Scheduling Scheme for Wireless Network Coding
}

\author{
Athanasios Gkelias \#1, Kin K. Leung \# \\ \# Electrical and Electronic Engineering Department, Imperial College Lodnon \\ South Kensington Campus, London SW7 2AZ, UK. \\ ${ }^{1}$ a.gkelias@imperial.ac.uk
}

\begin{abstract}
Network coding (NC) is an emerging technology area promising to increase the utilization of both wired and wireless networks. It can be seen as an extension to routing by allowing relay nodes to combine the information received from multiple links in the subsequent transmissions. NC was initially proposed in the context of multicast in wired networks where it was proven to achieve the maximum multicast capacity. However, the operation of $\mathrm{NC}$ in wireless networks with multiple unicast transmissions and its impact on the different layers has not been fully understood. In this paper we give a thorough insight into the interaction of $\mathrm{NC}$ and scheduling in wireless networks and we propose a novel distributed proportional fair (PF) scheduling algorithm that exploits multiuser diversity and coding opportunities to maximize the network throughput while guarantees "proportional fairness".
\end{abstract}

\section{INTRODUCTION}

Network Coding (NC) emerged less than a decade ago as a promising new technique to increase the utilization and throughput of wired and wireless networks [1]. In that seminal work authors shown that the multicast network capacity can always be achieved in any wired network if we allow intermediate nodes to not only forward but also combine and process the incoming information flows. Since then, $\mathrm{NC}$ has attracted increased research interest mainly from the information theoretical perspective. Although theoretical results demonstrate the potential advantages of NC (including bandwidth and power efficiency, computational efficiency and robustness to network dynamics and link failures [1],[2],[3]), there are only limited references on actual implementations of $\mathrm{NC}$ in real networks [4] where several practical issues spanning all protocol layers have to be solved or taken into account.

In this work we mainly concentrate on the implementation of $\mathrm{NC}$ in the medium access control (MAC) layer of wireless mesh-type networks. NC imposes several new challenges in the design of scheduling schemes. A node has the option to jointly encode its packets with other packets to increase the coding gain and overall network throughput. However, such packets may not be always available. This fact imposes a tradeoff between the network throughput gain and the packet delay that the underlying application can afford. Moreover, the broadcasting nature of the wireless channel may provide coding opportunities, on the other hand though, it may create some fairness problems between the encoded links. This is because the encoded packets have to be broadcasted with a rate limited by the worst link. In this case, "strong" links can not utilize their full capacity. Similar problem can occur because of the overhearing, where some nodes may have to sacrifice part of its own achievable link throughput and transmit their packets in a lower bit rate in order to allow more nodes (e.g., nodes with worst links) to capture their transmissions in order to increase the NC opportunities.

On top of this, wireless channel fluctuations are known to provide opportunities for multiuser diversity gain. However, as we demonstrate later in this paper, while channel fluctuations in wireless links can provide multiuser diversity gain, on the other hand they may decrease the overall network throughput when NC is improperly used. All of these issues have to be taken into account in the design of efficient network-coding aware scheduling algorithms that can maximize the overall network throughput while at the same time ensure fairness to all nodes and quality-of-service to the running applications.

Related Work: The interaction of NC and wireless MAC protocols has attracted limited research interest so far. Moreover, most of the proposed works focus on theoretical bounds/approaches, wired networks and sometimes unrealistic for real networks assumptions. Reference [5] introduces an opportunistic scheduling strategy. Authors assume that the transmission from individual nodes to a relay node (i.e., the encoder) as well as overhearing are performed ideally to create a network group. They only consider the scheduling by the relay node after it becomes ready to perform network coding. However, in wireless network overhearing has a great impact on the coding opportunities and network throughput as we will demonstrate in the following sections. In [6], authors utilize $\mathrm{NC}$ to provide an efficient MAC-layer retransmission scheme for single-hop wireless networks where many mobile stations are communicating with a single access point (AP). Authors in [7] demonstrate that $\mathrm{NC}$ should be jointly considered with scheduling to maximize the network capacity and provide a methodology to develop optimal and adaptive joint network coding and scheduling schemes. However, their analysis is based on the assumption that no opportunistic listening takes place in the network. This limits in their work the coding opportunities since a node can decode a packet consists of $m$ - 
XOR-ed packets i.f.f. this node has already transmitted $m-1$ of the encoded packets itself.

Our contribution: Our main contributions are summarized as follows: (1) We demonstrate the tradeoffs between the multiuser diversity gain and $\mathrm{NC}$ gain. (2) We show that in wireless networks, NC may degrade the overall network performance if channel information is not taken into account. (3) We design a distributed proportional fair NC aware scheduling scheme which, by taking two-hop scheduling decisions, exploits both the multiuser diversity gain and coding opportunities to maximize the overall wireless network throughput while guarantees fairness to the underlying data flows.

The remaining of this paper is organized as follows. In Section II, the underlying system model and its assumptions are thoroughly explained. The impact of wireless channel statistics on NC coding opportunities is discussed in Section III. A novel NC aware scheduling algorithm and its performance is demonstrated in Section IV. Finally, conclusions are drawn in Section V.

\section{SYSTEM MODEL}

We define as $\lambda_{i, j}$ the link throughput rate for the link $\ell_{i, j}$. Let $M_{i}^{\lambda_{0}}$ denote the number of nodes that can achieve communication rate with node $i$ higher or equal to $\lambda_{0}$ and let $\mathcal{M}_{i}^{\lambda_{0}}$ represent the set of these nodes, i.e., $\mathcal{M}_{i}^{\lambda_{0}}=\left\{m_{k}\right\}_{k=1}^{M_{i}^{\lambda_{0}}}$, $\lambda_{i, m_{k}} \geq \lambda_{0}$. It is obvious that, in a wireless channel, as $\lambda_{0}$ decreases, $M_{i}^{\lambda_{0}}$ increases since node $i$ can decode more distant users. We assume that every node $i$ can operate as central controller for all nodes $j \in \mathcal{M}_{\lambda_{0}}^{i}$. This means that node $i$ can operate either as a relay for any 2-hops transmitterreceiver pair within $\mathcal{M}_{\lambda_{0}}^{i}$, or as a network encoder, receiving flows from a group of transmitters and broadcasting the encoded information to the respective receivers within $\mathcal{M}_{\lambda_{0}}^{i}$. Let $\mathcal{S}_{i}^{\lambda_{0}}=\left\{s_{i}^{k}\right\}_{k=1}^{S_{i}^{\lambda_{0}}}$ denote the set of $S_{i}^{\lambda_{0}}$ feasible coding or scheduling decisions taken by node $i$. For the link scheduling, time division multiple access (TDMA) is considered where the slot duration is set to $t_{0}$. Finally, let $B_{i, j}$ denote the buffer that corresponds to the flow from node $i$ to node $j$, where $i$ and $j$ are 2-hops neighbors, and let $b_{i, j}$ represents the amount of data in buffer $B_{i, j}$.

Effective Group Throughput: We define as effective group throughput $R_{s_{i}^{k}}$, the joint throughput that can be achieved in $t_{0}$ by the nodes belong to $\mathcal{M}_{i}^{\lambda_{0}}$ if the $s_{i}^{k} \in \mathcal{S}_{i}^{\lambda_{0}}$ decision is taken by node $i$.

In the following we give two examples (Fig.1) on how the group throughput is calculated if the central controller performs (a) simple relay and (b) network coding in. Example 1 (Relaying): Consider the wireless network in Fig.1(a) where node $c$ operates as a relay for the communication of nodes $a$ and $e$ (i.e., $s_{c}:\{a \rightarrow c, c \rightarrow e\}$ ). Let $t_{i, j}$ denote the time slot allocated to link $\ell_{i, j}$ while the time allocated to $s_{c}$ is the slot duration $t_{0}$ (for simplicity, $t_{0}$ is normalized to unity). In order to avoid a bottleneck in this route $c$ has to allocate the time resources such that $\lambda_{a, c} t_{a, c}=\lambda_{c, e} t_{c, e}$ where $t_{a, c}+t_{c, e}=t_{0}=1$. However, the amount of data in buffer

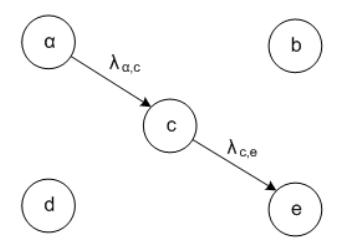

(a)

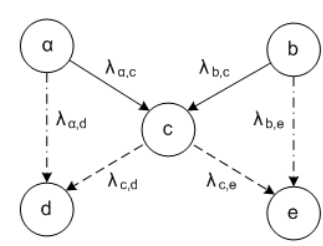

(b)
Fig. 1. Communication scenario examples for central controller $c$ : (a) relaying (b) coding.

$B_{a, e}$ may be less than the amount of data that node $c$ is able to relay in $t_{0}$, or in other words, $b_{a, e}<\lambda_{a, c} t_{a, c}$. Therefore the effective group throughput for the decision $s_{i}$ is calculated as:

$$
R_{s_{i}}=2 \min \left(b_{a, d}, \frac{\lambda_{a, c} \lambda_{c, e}}{\lambda_{a, c}+\lambda_{c, e}}\right)
$$

Example 2 (Network Coding): Consider now the communication scenario in Fig.1(b) where nodes $a$ and $b$ want to communicate with nodes $e$ and $d$ respectively, and node $c$ operates as central controller, and let $f_{i}$ denote the information flow transmitted from node $i$. If network coding is considered, node $c$ can take advantage of the coding opportunity that arises in the group, i.e., $d$ and $e$ are able to overhear the transmissions from nodes $a$ and $b$ respectively. Therefore, $c$ can jointly encode the flows $f_{a}$ and $f_{b}$ by using the XOR operation and broadcast the encoded flow $f_{a \oplus b}$. Node $e(d)$ has already overheard the flow $f_{b}\left(f_{a}\right)$ and can obtain $f_{a}\left(f_{b}\right)$ from $f_{a \oplus b}$. Therefore the coding decision $s_{i}:\{a \rightarrow c, b \rightarrow c, c \rightarrow(d, e)\}$ is chosen. However now, because of the overhearing the achievable rate for the transmission from node $a(b)$ will be $\lambda_{a,(c, d)}=\min \left\{\lambda_{a, c}, \lambda_{a, d}\right\} \quad\left(\lambda_{b,(c, e)}=\min \left\{\lambda_{b, c}, \lambda_{b, e}\right\}\right)$. Moreover, since the encoded flow $f_{a \oplus b}$ has to be broadcast to both $d$ and $e$ the achievable rate for the transmission from node $c$ will be $\lambda_{c,(d, e)}=\min \left\{\lambda_{c, d}, \lambda_{c, e}\right\}$. Again, in order to avoid bottlenecks in the flows, node $c$ has to allocate the time resources such that $\lambda_{a,(c, d)} t_{a,(c, d)}=\lambda_{b,(c, e)} t_{b,(c, e)}=$ $\lambda_{c,(d, e)} t_{c,(d, e)}$ and $t_{a,(c, d)}+t_{b,(c, e)}+t_{c,(d, e)}=t_{0}=1$. Taking into account the buffer queues $b_{a, e}$ and $b_{b, d}$ at transmitters $a$ and $b$ respectively, the effective group throughput for the given coding decision can be easily calculated as:

$$
R_{s_{i}}=4 \min \left(b_{a, e}, b_{b, d}, R_{s_{i}}^{\prime}\right)
$$

where

$$
R_{s_{i}}^{\prime}=\left(\frac{1}{\lambda_{a,(c, d)}}+\frac{1}{\lambda_{b,(c, e)}}+\frac{1}{\lambda_{c,(d, e)}}\right)^{-1}
$$

\section{Wireless Network COding Opportunities}

From equations (1) and (2) someone can observe that although NC provides coding gain, may not always increase the overall network throughput. For instance, in the last example, $\mathrm{NC}$ provides a coding gain of $4 / 3$ compare to conventional 
scheduling (i.e., 3 transmissions are required with NC while scheduling requires 4 transmissions). However, if $\lambda_{a, d}\left(\lambda_{b, e}\right)$ is smaller than $\lambda_{a, c}\left(\lambda_{b, c}\right)$ then node $a(b)$ has to reduce its transmission rate in the direct link $\ell_{a, c}\left(\ell_{b, c}\right)$ so that $d(e)$ will be able to overhear its transmission. This has as a result, if the overhearing links are much worst than the direct links, the effective group throughput to decrease despite the coding gain that NC provides.

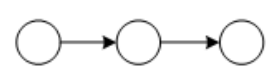

(a)

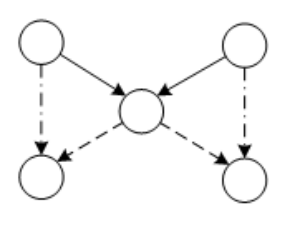

(c)

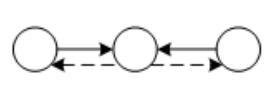

(b)

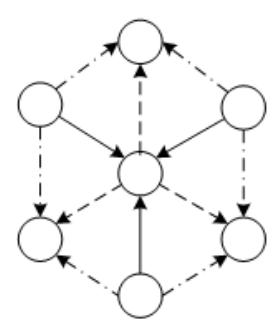

(d)
Fig. 2. Scheduling-Coding scenarios: (a) simple relay (b) 2-nodes NC without overhearing (c) 4-nodes NC with overhearing (d) 6-nodes NC with overhearing.

Moreover, in wireless networks, channel statistics must be taken into account. Multipath scattering from objects near the antenna causes rapid fluctuations of the received signal power, whereas shadowing by dominant obstacles is experienced as a slow amplitude affect. It is well known that such fluctuations can provide multiuser diversity gain, i.e., the network throughput is maximized by allowing transmission in the link with the maximum channel gain each time. However, if $\mathrm{NC}$ is used, due to the multicast requirements, the data rate of each multicast channel is limited by the link with the minimum channel gain.

In order to have a better understanding of this tradeoff between multiuser diversity gain and NC gain the following network simulation scenario is considered. Nodes are uniformly distributed in a two-dimensional space in a way that each node has 6 one-hop equally distant neighbors. In this way all communication links will suffer the same path loss. We assume that each link fluctuates independently according to a $\log$ normal distribution (i.e., shadowing). Figure 2 depicts all the considered communication (coding or scheduling) scenarios.

Table I demonstrates the percentage of time that the different $\mathrm{NC}$ or scheduling scenarios depicted in Figure 2 are the optimum decisions (i.e., higher effective group throughput) for different channel conditions. For instance, for log-normal shadowing with mean $0 \mathrm{~dB}$ and $\sigma=6 \mathrm{~dB}, 88.58 \%$ of the time is preferable to simple schedule (Fig.2(a)) rather than encode packets. On the other hand for mean $10 \mathrm{~dB}$ and $\sigma=0 \mathrm{~dB}$, almost $100 \%$ of the time the channel gives the opportunity of 3 transmitters to jointly encode their packets (i.e. 2(d)). It is interesting to observe and note here that while high channel variations (i.e., big $\sigma$ ) traditionally increase the multiuser diversity gain, however, they decrease the coding opportunities in the network and vice versa. Since NC has been introduced as a layer 3 technique, our results show that if PHY layer information is ignored (e.g., always use NC independently of the channel statistics) NC may decrease the overall network throughput. Moreover, there are couple more issues that has to be taken into account:

TABLE I

CODING OPPORTUNITIES FOR DiFFERENT CHANNEL CONDITIONS

\begin{tabular}{|l|l|l|l|l|l|}
\hline \hline$m$ & $\sigma_{d B}$ & $(\mathrm{a})$ & $(\mathrm{b})$ & $(\mathrm{c})$ & $(\mathrm{d})$ \\
\hline $0 \mathrm{~dB}$ & $0 \mathrm{~dB}$ & $57.20 \%$ & $22.48 \%$ & $20.30 \%$ & $0.02 \%$ \\
\hline $0 \mathrm{~dB}$ & $3 \mathrm{~dB}$ & $79.31 \%$ & $11.25 \%$ & $9.43 \%$ & $0.01 \%$ \\
\hline $0 \mathrm{~dB}$ & $6 \mathrm{~dB}$ & $88.58 \%$ & $6.50 \%$ & $4.92 \%$ & $\approx 0 \%$ \\
\hline $5 \mathrm{~dB}$ & $0 \mathrm{~dB}$ & $0.30 \%$ & $48.49 \%$ & $41.55 \%$ & $9.66 \%$ \\
\hline $5 \mathrm{~dB}$ & $3 \mathrm{~dB}$ & $13.68 \%$ & $45.02 \%$ & $40.74 \%$ & $0.56 \%$ \\
\hline $5 \mathrm{~dB}$ & $6 \mathrm{~dB}$ & $48.86 \%$ & $26.62 \%$ & $24.45 \%$ & $0.07 \%$ \\
\hline $10 \mathrm{~dB}$ & $0 \mathrm{~dB}$ & $\approx 0 \%$ & $\approx 0 \%$ & $\approx 0 \%$ & $100 \%$ \\
\hline $10 \mathrm{~dB}$ & $3 \mathrm{~dB}$ & $\approx 0 \%$ & $12.07 \%$ & $5.31 \%$ & $82.62 \%$ \\
\hline $10 \mathrm{~dB}$ & $6 \mathrm{~dB}$ & $0.04 \%$ & $46.80 \%$ & $38.68 \%$ & $14.48 \%$ \\
\hline
\end{tabular}

Fairness: Even if higher overall network throughput is achieved, in many cases, some links may have to sacrifice part of their own throughput. This is due to the fact that all incoming to the encoder data flows must limit their link throughput to be lower than the throughput of the outgoing encoded flow. Moreover, the broadcast throughput of the encoded flow is limited by the lower capacity of the involved unicast links. This means that some links may have to transmit in much lower throughput than they are actually capable of. This may affect the proper functionality of higher layers, such as routing algorithms, especially if quality-of-service (QoS) is considered.

Delay: Data flows towards specific routes may not be always available or some buffers may not have enough amount of data for joint coding. Therefore, some nodes may delay their transmissions anticipating flows towards routes that can generate NC opportunities or their neighbors to buffer more data for joint coding. This situation works fine for data flows without delay constraints, since the overall network throughput will be maximized. However, for delay critical applications this delay can be proven catastrophic. Moreover, such delays may also have unpredictable effect on the performance of the TCP protocol since those delays may wrongly be interpreted as network congestions.

All these aforementioned issues must be considered when new network coding aware protocols for wireless networks are designed.

\section{NC AWARE SCHEDUling Algorithm}

In order to exploit the NC opportunities while taking into account all the aforementioned issues we propose a novel opportunistic proportional fair NC aware scheduling scheme for wireless networks. Traditional opportunistic scheduling algorithms take decisions based only on favorable wireless link conditions. However, if NC is introduced, the scheduler 
has to also decide the best group of links that will be jointly coded. At the same time it must maintain fairness in terms of resource efficiency and delay to all the users.

The main idea is that each node tries to become central controller for all its neighbor nodes (i.e., one-hop away nodes). To achieve this, it has to propose its own scheduling/NC scheme and compete with the others. The node with the best proposed scheme will become central controller in its neighborhood for the frame duration after which the nodes will compete again. In the following we describe in more details the proposed algorithm.

\section{A. Signaling}

We assume that a separate low bandwidth channel is used for signaling. The signaling information to be exchanged between the nodes described in the following.

1) All nodes measure periodically the link quality with all their one-hop neighbors by exchanging pilot symbols.

2) All nodes check their routing tables and identify 2-hops network coding opportunities assuming that they are the encoders.

3) All node estimates the set of all their scheduling (relay) and coding decisions.

4) For all coding decisions, nodes ask all other nodes involved in the corresponding coding decisions for their multicast throughput. (For instance, in Fig.1(b) node $c$ identify the following scheduling $s_{c}^{1}:\{a \rightarrow c, c \rightarrow e\}, s_{c}^{2}:\{b \rightarrow c, c \rightarrow d\}$ and coding $s_{c}^{3}:\{a \rightarrow c, b \rightarrow c, c \rightarrow(d, e)\}$ decisions. Then asks nodes $a$ and $b$ for $\lambda_{a,(c, d)}$ and $\lambda_{b,(c, e)}$ respectively.)

5) Each node calculates the effective group throughput for every coding/scheduling decision based on (1) and (2) and broadcasts to its neighbors the one with the higher utility described in the following. Only the utility value is transmitted, not the scheduling/coding decision.

6) All nodes retransmit this values, such that, the utility value of each node $i$ will be received by all nodes within two-hops radius from $i$.

7) After all nodes receive the utility values from all their 1-hop and 2-hops neighbors, the one with the higher utility becomes the central controller in its 2-hops neighborhood for the next frame.

\section{B. Utility Function:}

Let $H_{i, j}[n]$ denote the estimate of the average running throughput for link $\ell_{i, j}$ (where $i$ and $j$ are 2-hop neighbors) at time $n$, averaged using an exponentially weighted low pass filter parameterized by $h_{o}$, which is a free parameter of the scheduler design. Each node keeps a track of the average throughput of all incoming and outgoing links over $h_{o}$ frames and updates their value as:

$H_{i, j}[n+1]=\left\{\begin{array}{cl}\left(1-\frac{1}{h_{o}}\right) H_{i, j}[n]+\frac{R_{s_{c}}[n]}{h_{o} L} & \text { if } \ell_{i, j} \in s_{c}^{k} \\ \left(1-\frac{1}{h_{o}}\right) H_{i, j}[n] & \text { if } \ell_{i, j} \notin s_{c}^{k}\end{array}\right.$

where $H_{i, j}[0]=\delta_{i, j}$ is some initial value, $R_{s_{c}^{k}}$ is the group throughput defined in Section II for the $s_{c}^{k}$ scheduling/NC decision taken by node $c$, and $L$ is the number of links involved in the scheduling/NC decision $s_{c}^{k}$. From the set of $\mathcal{S}_{c}^{\lambda_{0}}$ the scheduler $c$ in frame $n$ will choose the $k$-th scheduling/NC decision $s_{c}^{k}$ that:

$$
s_{c}^{k}=\arg \max _{s_{c}^{k} \in \mathcal{S}_{c}^{\lambda_{0}}}\left\{\frac{R_{s_{c}^{k}}[n]}{\min _{\ell_{i, j} \in s_{c}^{k}}\left\{H_{i, j}[n]\right\}}\right\}
$$

As discussed before in the signaling subsection, each node $i$ will broadcasts to its one-hop and two-hops neighbors the calculated by (5) value $G_{i}$ of the chosen $k$-th scheduling/NC decision:

$$
G_{i}=\frac{R_{s_{i}^{k}}[n]}{\min _{\ell_{i, j} \in s_{i}^{k}}\left\{H_{i, j}[n]\right\}}
$$

After all nodes receive the utility values from all their onehop and two-hops neighbors, the node $i$ with the higher utility, i.e, $i=\arg \max \left\{G_{i}[n]\right\}$ becomes the central controller within its two-hops neighborhood for the next frame. With these decisions, the best scheduling/NC decision that maximizes the group throughput is taken while the average links throughput remains reasonably fair.

Note here that in traditional proportional fairness scheduling decisions (e.g., for cellular systems) fairness is provided per user/terminal. However, if $\mathrm{NC}$ is considered, the coding decisions involve cooperation between group of nodes and the amount of data that a node transmits may not correspond to its own traffic. If fairness is provided per node basis the "good links" of a node will be served, the average running throughput, given by (4), of this node will increase, while the "bad links" of the same node will starve. Moreover, since we consider two-hop scheduling/NC decisions, the same link may be part of several flows ending up to different receivers. Therefore, providing fairness per link basis can be proven unfair for some flows. This is the reason why we have considered separate buffers for every 2-hops flows and 2-hop link fairness. In addition, separate buffers per flow will also decrease the computational complexity since a node does not have to search a single buffer for the appropriate packets for encoding.

\section{Simulation Results}

In our simulations the following system model has been considered. The network topology is the same as in Section III. Nodes have half-duplex transceivers (and they all transmit at the same fixed power. Nodes generate constant data rate unicast flows for all their 2-hop neighbors and separate buffers are used for each flow. Although, the data flows have a constant rate, the queue size in each buffer varies due to the channel variations and scheduling/NC decisions. The scheduling/NC decisions are taken at the beginning of each time slot and the wireless channel is assumed to remain constant for the whole slot duration. For the wireless channel, only largescale propagation effects are considered, i.e., path-loss and log-normal shadowing. We assume that the channel statistics are independent for each wireless link. As for the signalling 


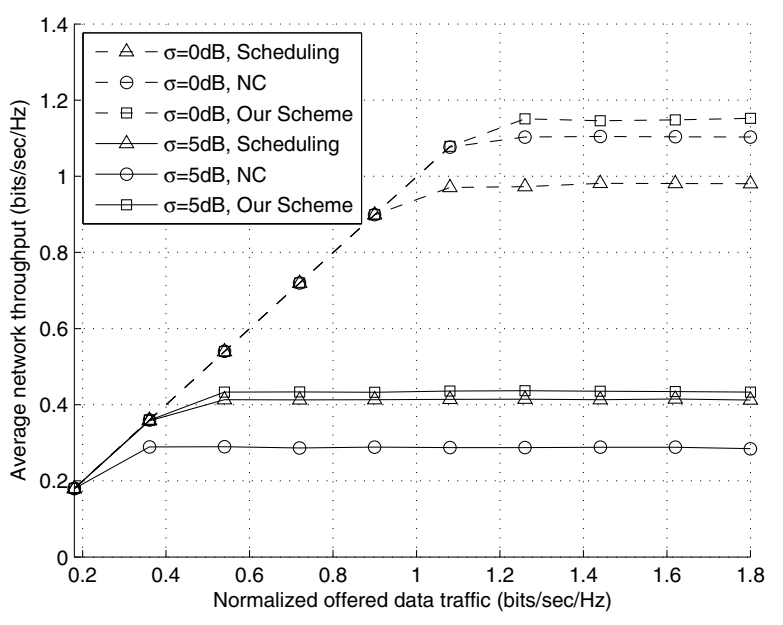

Fig. 3. Average network throughput in bits/sec/Hz vs. the normalized offered data traffic for different shadowing parameters $(\mathrm{SNR}=5 \mathrm{~dB})$ and for differen scheduling/coding scenarios.

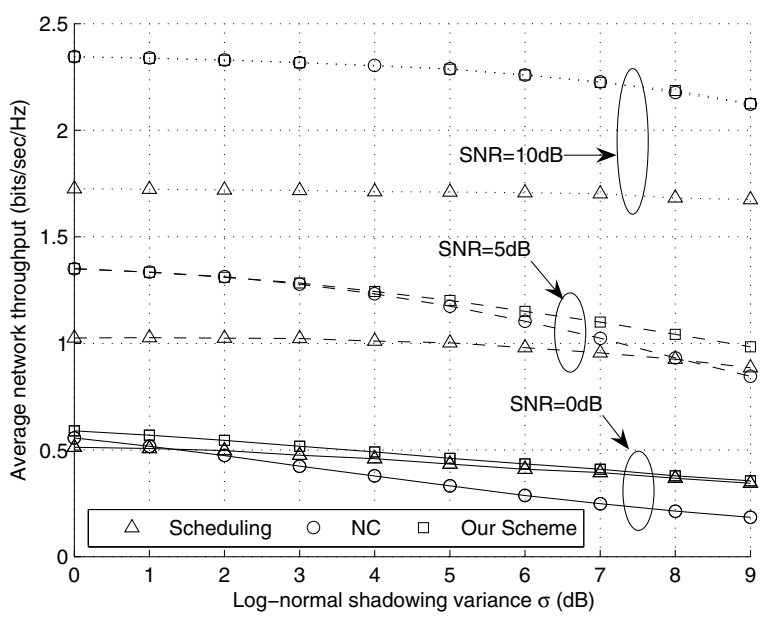

Fig. 4. Average network throughput in bits/sec/Hz vs. Log-normal shadowing variance $\sigma_{d B}$ for different values of SNR and scheduling/coding scenarios (full buffers).

exchange, we consider a separate low bandwidth, errorless channel.

Figure 3 depicts the performance of the proposed algorithm in terms of the achievable average network throughput (in bits/sec/Hz) as a function of the offered data traffic (the incoming traffic has been normalized over the used channel bandwidth). The performance is compared with the cases where (1) only scheduling and (2) only NC decisions are taken. As it can be observed, for signal-to-noise-ratio (SNR) of $0 \mathrm{~dB}$ and for low shadowing variance $(\sigma=0 \mathrm{~dB})$, the "only coding" scheme outperforms the "only scheduling" scheme. However, for higher variance $(\sigma=5 \mathrm{~dB})$, the observation is exactly the opposite. Nevertheless, in both case our proposed scheme outperforms the other two schemes, guaranteeing higher network throughput.
In Figure 4, the impact of the receiver SNR and the variance $\sigma_{d B}$ of the log-normal shadowing on the proposed scheme is demonstrated. All buffers in this case are considered full. It is clear that higher SNR increases the throughput achieved by $\mathrm{NC}$ decisions as compared to scheduling decisions. However, that throughput decreases as $\sigma_{d B}$ increases, while for low SNR and high $\sigma_{d B}$ scheduling provides higher throughput than coding. This is because of the limitations imposed by overhearing and broadcasting as they have been discussed in Section II. Again our proposed scheme, by successfully combining both decisions always provides higher network throughput independent of the channel conditions.

\section{CONCLUSIONS}

In this paper we gave an insight into several practical issues related to the interaction of $\mathrm{NC}$ and scheduling in wireless networks and we demonstrated the tradeoff between multiuser diversity gain and $\mathrm{NC}$ gain. We showed that if $\mathrm{NC}$ is improperly used, it may result to reduction of the overall wireless networks throughput. Moreover, there are several fairness and delay issues that have to be considered in the design of NC aware wireless protocols. By taking into account some of these practical issues we proposed a novel distributed proportional fair NC aware scheduling scheme that exploits the coding opportunities appearing in wireless networks to maximize the overall network throughput while guarantees the required fairness to the underlying network flows. The impact of different channel statistics and random network topologies on the performance of the proposed algorithm will be part of our future work.

\section{ACKNOWLEDGMENTS}

The work reported in this paper has formed part of the Flexible Networks area of the Core 5 Research Programme of the Virtual Centre of Excellence in Mobile \& Personal Communications, Mobile VCE, www.mobilevce.com, and has been jointly funded by Mobile VCE's industrial member companies and the UK Government, via the Engineering and Physical Sciences Research Council.

\section{REFERENCES}

[1] R. Ahlswede, N. Cai, S.-Y. Li, and R. Yeung, "Network information flow," IEEE Trans. Inf. Theory, vol. 46, no. 4, pp. 1204-1216, Jul. 2000.

[2] Y. Sagduyu and A. Ephremides, "Some optimization trade-offs in wireless network coding," in Proc. CISS'06, Mar. 2006, pp. 6-11.

[3] T. Ho, R. Koetter, M. Mèdard, D. Karger, and M. Effros, "The benefits of coding over routing in a randomized setting," in Proc. IEEE ISIT'03, Jun./Jul. 2003, p. 442.

[4] S. Katti, H. Rahul, W. Hu, D. Katabi, M. Mèdard, and J. Crowcroft, "Xors in the air: Practical wireless network coding," IEEE/ACM Trans. Netw., vol. 16, no. 3, pp. 497-510, Jun. 2008

[5] H. Yomo and P. Popovski, "Opportunistic scheduling for wireless network coding," in Proc. IEEE ICC'07, Jun. 2007, pp. 5610-5615.

[6] F.-C. Kuo, K. Tan, X.-Y. Li, J.Zhang, and X. Fu, "Network codingaware fair opportunistic scheduling in lossy wireless networks," Institute of Computer Science, University of Goettingen, Goettingen, Germany, Technical Report IFI-TB-2008-003, 2008.

[7] P. Chaporkar and A. Proutiere, "Adaptive network coding and scheduling for maximizing throughput in wireless networks," in Proc. MOBICOM'07, Sep. 2007, pp. 135-146. 